\title{
EDITORIAL
}

\section{Introduction to Special Section on Advances of Orange Technologies}

\author{
Lei XIE $(\bowtie)^{1}$, Jhing-Fa WANG ${ }^{2}$ \\ 1 School of Computer Science, Northwestern Polytechnical University, Xi' an 710072, China \\ 2 Department of Electrical Engineering, Cheng Kung University, Tainan 701, China
}

(c) Higher Education Press and Springer-Verlag Berlin Heidelberg 2017

Orange technology, together with green technology, forms the double helical structure of sustainable human development. Instead of emphasizing the relations between environments and humans, as proposed by green technology, the orange technology aims to discover the relationship between human development and happiness, and bring more health, happiness, warming care, and more mental wellness to the society. The research scope is definitely multidisciplinary, which includes computer science, electrical engineering, biomedical engineering, psychological/physiological science, cognitive science, social science, and dechnology (design + technology).

This special section aims to bring together researchers and technologists engaged in this emerging area and discuss some of the latest developments. Three articles are finally accepted after strict peer reviews.

It is usually hard to interpret human speech and to detect human emotion status in real-world scenarios as speech may be contaminated with unwanted noises. Beamforming using microphone array is widely used to solve this problem as it offers better spatial focusing capability than single microphone. However, there always exists a trade-off between the directivity capability of an array and its robustness on system errors. The first article, entitled "Constrained minimum power combination for broadband beamformer design in the STFT domain", proposes a constrained minimum-power combination method to combine the merits of different beamformers rather than trade off their performances.

Warming care is an active area in orange technology. Better human hearing technologies, such as hearing aids and immersive auditory, have increasingly drawn attentions recently. Virtual 3D audio, which aims to place sound sources anywhere in threedimensional space, has many practical and potential applications in healthcare. The second article in this special section, entitled "Sound image externalization for headphone based real-time 3D audio", tries to improve the 3D audio effects by a better sound image externalization approach.

Affective computing for happiness detection is another key research area in orange technology. As we know, human speech reflects speaker's emotion status. However, it is a challenging task to detect the emotion status (specifically happiness/suffering factors) from spoken dialogues because conversational speech is difficult to interpret. The last article of this special section, entitled "Spoken dialog summarization system with HAPPINESS/SUFFERING factor recognition", proposes an integrated system that can simultaneously summarize human spoken dialogues from speech recognition transcripts meaningfully and predict the happiness/suffering factor from those dialogues accurately.

Orange technology is an emerging multidisciplinary field with wide research topics. Due to the space limit, this special section only focuses on a bit of the latest developments. We hope that the readers will find these articles informative and interesting.

Received April 25, 2017

E-mail: 1xie@nwpu.edu.cn; wangjf@csie.ncku.edu.tw 